\title{
Some Constraints on The Formation of Globular Clusters
}

\author{
Keith M. Ashman \\ Department of Physics and Astronomy, University of Kansas, Lawrence, KS 66045; and Department of Physics, \\ Baker University, Baldwin, KS 66006 \\ e-mail: ashman@kuspy.phsx.ukans.edu \\ Stephen E. Zepf \\ Department of Astronomy, Yale University, New Haven, CT 06520 \\ e-mail: zepf@astro.yale.edu
}

\begin{abstract}
We explore the constraints on globular cluster formation provided by the observed conditions in starbursts where globulars are currently forming, and by the observed properties of young and old globular clusters. We note that the pressure in the ISM of starbursts and mergers implies that molecular clouds in these environments have radii similar to those of globular clusters. Such molecular clouds are therefore viable precursors to globular clusters if the star formation efficiency in the clouds is high. A high star formation efficiency may be a consequence of the high density and associated high binding energy and short dynamical timescale of molecular clouds in such environments. We also note that the apparent lack of a mass-radius relationship in young and old globular cluster systems places important constraints on globular cluster formation models. This is because molecular clouds are observed to follow a virial scaling relation between mass and radius. We suggest that a variable star formation efficiency may weaken or eliminate the mass-radius relation of molecular clouds as they fragment to form globular clusters. We attribute the absence of young globular clusters in the disks of ordinary galaxies such as the Milky Way to the relatively low ambient pressures in such systems.
\end{abstract}

Subject headings: globular clusters: general — stars: formation 


\section{Introduction}

Globular clusters are found around nearly all galaxies, from low-luminosity dwarf spheroidals to the most massive elliptical galaxies. However, globular clusters are not currently forming in any significant numbers in the disks of ordinary galaxies like the Milky Way. Models of globular cluster formation must therefore address two related questions. First, what physical processes are responsible for the formation of dense, massive star clusters in a broad range of environments? Second, why is globular cluster formation common in starbursts, but rare in the star formation regions of disk galaxies?

The absence of young globular clusters in the Milky Way has compounded the difficulty in understanding globular cluster formation. Indeed, until relatively recently, globular clusters were often viewed as being exclusively old objects. As a result, most globular cluster formation models have been divorced from direct observation of star cluster formation and have instead been based on ideas concerning the physical conditions at the pregalactic and protogalactic epochs. For example, Peebles and Dicke (1968) suggested that globular clusters are the first bound structures to form in the universe and therefore predate galaxies. Subsequent hierarchical clustering ensures that at least some of the original population of globulars end up in the halos of galaxies. Such "primary" formation models have fallen from favor for a variety of reasons. These include the presence of a thick disk population of metal-rich globular clusters in the Milky Way (Zinn 1985 and references therein), correlations between the location of globular clusters in their parent galaxies and globular cluster properties such as metallicity (e.g. Harris 1991), and the absence of dark matter halos around globular clusters (Moore 1996) that would be expected if these objects had a cosmological origin (see Ashman and Zepf 1998 for a detailed discussion).

The above considerations moved the focus of globular cluster formation models to the protogalactic epoch. One influential model is due to Fall \& Rees (1985) who suggested that protoglobular clouds form through thermal instability in protogalaxies. Fall \& Rees (1985) argued that such clouds would cool to around $10^{4} \mathrm{~K}$. At this temperature, cooling in a primordial (very low-metallicity) plasma becomes inefficient under equilibrium conditions, in which case the clouds "hang up" at $10^{4} \mathrm{~K}$. The Jeans mass of such clouds is around $10^{6} M_{\odot}$ which is in the same ballpark as the median mass of Milky Way globular clusters. Similar scenarios were proposed by Gunn (1980), McCrea (1982) and Murray \& Lin (1992).

Further investigation of this problem has shown that it is extremely difficult to maintain protoglobular clouds at a constant temperature for a sufficient time that a characteristic mass is imprinted on an initial cloud mass spectrum. Even in a primordial plasma non-equilibrium cooling can generate significant amounts of $\mathrm{H}_{2}$ which provides additional cooling (Ashman 1990 and references therein). Moreover, the high metallicity of many globular clusters, such as the metal-rich population found in elliptical galaxies and the thick disk clusters of the Milky Way, suggests that their precursor clouds were pre-enriched with metals. The presence of such metals allows clouds to cool efficiently well below $10^{4} \mathrm{~K}$. The dependence of cooling rate on metallicity also means that Fall-Rees type models predict that the mass of globular clusters should decrease with increasing globular cluster metallicity. Such a trend is ruled out observationally (e.g. Djorgovski \& Meylan 1994). Further, the physical conditions required by thermal instability models do not exist in environments where globular clusters currently form, nor would they have ever existed in the lowest mass galaxies with globular cluster systems (Ashman \& Zepf 1998).

Ideas concerning globular cluster formation have been greatly advanced by HST observations of young star clusters in galaxy starbursts and mergers (e.g., Schweizer 1998; Ashman \& Zepf 1998). Specifically, HST images reveal that widespread formation of compact, bright, blue star clusters is a characteristic feature of vigorous starbursts. These young star clusters have sizes and estimated masses like those of old Galactic globular clusters. The one aspect of the young globular cluster systems that is different than that of old systems is their inferred mass functions. Old globular cluster systems are well-known to have a log-normal mass function, while the mass function inferred for young systems is consistent with a single power law over a range that includes the mass scale at which the old cluster systems begin to decline in numbers (Whitmore et al. 1999; Zhang \& Fall 1999; Zepf et al. 1999; Carlson et al. 1998; Miller et al. 1997). However, since destructive processes are expected to preferentially remove low-mass clusters from the young cluster population, these observations are consistent with the idea that systems of young 
clusters in starbursts will evolve to resemble the traditional old systems of globular clusters (e.g. Fall \& Rees 1977; Murali \& Weinberg 1996; Gnedin \& Ostriker 1997; Vesperini 1997).

It has been pointed out by several authors (e.g., Elmegreen \& Efremov 1997) that the mass function slope of Giant Molecular Clouds (GMCs) in the Milky Way and other galaxies is similar to that of young globular clusters in mergers. Given that GMCs are known to be the sites of open cluster formation, it is reasonable to suppose that similar molecular clouds are the precursors of young globular clusters. The similarity in mass functions adds weight to this idea. However, the star formation efficiency in GMCs in galaxies like the Milky Way is only around $0.2 \%$. For the typical masses of such GMCs, this efficiency produces open clusters with masses up to $10^{3} M_{\odot}$ or so. Thus in order to produce clusters spanning the full mass range of observed globular clusters, we either require more massive GMCs (Harris \& Pudritz 1994) or we require a much higher star formation efficiency.

In this paper, we present an analysis of the globular cluster formation process based on observations of the physical conditions in which globular clusters currently form, as well as observations of young globular clusters themselves. Specifically, we consider the conditions in the ISM of merger-induced starbursts and the properties of young globular clusters in this environment. Our results indicate that GMCs in starbursts are viable globular cluster progenitors, but that the mass-radius relation of young globular clusters places stringent constraints on the details of the globular cluster formation process. For GMCs with a power-law mass distribution like that in the Milky Way, the high pressure that is characteristic of the ISM in starbursts compresses GMCs so that they have the appropriate radii to become globular clusters, while the high star formation efficiency found in starbursts produces much more massive, tightly bound clusters than those formed in GMCs in the Milky Way. However, the lack of a significant massradius relation for young globular clusters (Zepf et al 1999), [also found in systems of open clusters (e.g. Testi, Palla \& Nota 1991; van den Bergh 1991) and old globular clusters (van den Bergh et al 1991; Djorgovski \& Meylan 1994; Ashman \& Zepf 1998)] is surprising, since any system of virialized gas clouds is expected to have a strong mass-radius relation. Thus a critical constraint on globular cluster formation models is that the mass-radius relation of precursor clouds must be eliminated. We show in this paper that a star formation efficiency that varies with the binding energy of the precursor clouds can wipe out a virial mass-radius relation.

In Section 2 we summarize the observational properties of systems of GMCs and globular clusters. We show in Section 3 that GMCs in the high-pressure ISM of starbursts are promising globular cluster precursors and discuss physical mechanisms that may lead to a high star formation efficiency in such clouds. Section 4 addresses the difference between the massradius relation of GMCs and globular clusters and the possibility that this difference may be the result of a star formation efficiency that varies with cloud binding energy. In Section 5 we present our conclusions.

\section{Observational Background}

In this section we describe the observed properties of GMCs and of young and old globular clusters. These properties must be incorporated into any model of globular cluster formation in which GMCs are globular cluster progenitors. Specifically, we discuss the mass and radius distributions of GMCs and globular clusters and the mass-radius relations followed by these objects. We review the evidence for the similarity at high masses of the GMC and globular cluster mass distributions. In contrast, the mass-radius relations followed by these objects differ. We also compare the radius distributions of globular clusters and GMCs. As we show in Section 4, these observations place strong constraints on any scenario in which globular clusters form from molecular clouds.

\subsection{The mass-radius relation of clouds and clusters}

A large body of observational evidence indicates that GMCs in a range of environments follow a massradius relation that is consistent with these objects being in virial equilibrium. Specifically, GMCs follow the scaling relation $r_{c} \propto M_{c}^{1 / 2}$ (Larson 1981), where $M_{c}$ is the cloud mass and $r_{c}$ is the cloud radius. This has been established for GMCs in the Milky Way (see the summary of observations given by Harris \& $\mathrm{Pu}-$ dritz 1994), as well as M33, the LMC and the SMC (Wilson \& Scoville 1990; Johansson 1991; Rubio et al. 1993).

In sharp contrast to GMCs, globular clusters appear to have a weak or non-existent correlation between radius and mass. This is relatively well-established 
for the Galactic globular cluster system (van den Bergh et al 1991; Djorgovski \& Meylan 1994; Ashman \& Zepf 1998) and seems to hold for the young globular cluster system in the merger/starburst NGC 3256 (Zepf et al. 1999), where there may be a shallow correlation. A similar weak or absent correlation between mass and radius also appears to hold for the young star clusters in the LMC (van den Bergh 1991), and for young star clusters in the Galaxy (e.g. Testi, Palla, $\&$ Nota 1999). In order to wipe out the mass-radius relation of the parent GMCs, it is apparent that either the mass or the radius (or both) of the final star clusters must differ from those of the original clouds. This difference in the mass-radius relations provides a challenge for any model in which globular clusters form from GMCs in virial equilibrium, particularly given the similarities in mass and radius distributions between GMCs and globular clusters discussed below. We return to this point in Section 4 and discuss how the difference might arise.

\subsection{The mass and radius distributions of GMCs and globular clusters}

The mass distribution of GMCs is usually expressed in the form:

$$
N\left(M_{c}\right) d M_{c} \propto M_{c}^{-\beta} d M_{c} .
$$

Published values of $\beta$ vary from study to study. Harris \& Pudritz (1994) summarize results from CO surveys in which the mass distribution of Milky Way GMCs is derived from the observed distribution of cloud sizes and the observed relation between cloud velocity dispersions and sizes (essentially a mass-radius relation). The result is $\beta=1.63 \pm 0.12$. A similar relation is found for clouds in M33, and for the LMC and SMC. Elmegreen \& Falgarone (1996) analyze more recent results and obtain $\beta$ in the range 1.5-2. However, they also emphasize the problem of systematic errors in mass measurement and suggest that without such errors the value of $\beta$ may be close to 2 in a wide range of environments.

The mass function of clusters may be written in a similar fashion:

$$
N\left(M_{*}\right) d M_{*} \propto M_{*}^{-\alpha} d M_{*},
$$

where, for young clusters, $\alpha \simeq 1.8$ (e.g. Whitmore et al. 1999; Zhang \& Fall 1999; Zepf et al. 1999; Carlson et al. 1998; Miller et al. 1997). The similarity between the mass function slope of GMCs and young globular clusters has been noted by several authors (e.g.,
Elmegreen \& Efremov 1997) and is an important motivation for our identification of GMCs as globular cluster progenitors. This value is also consistent with the high-mass end of the mass function of old globular clusters (e.g., Harris \& Pudritz 1994), with the possible exception of the most massive clusters (e.g. Burkert \& Smith 2000 and references therein). The fact that GMCs follow a mass-radius relation means that their mass and radius distributions are not independent quantities. We can express the GMC radius distribution in a manner analogous to the mass distributions given above:

$$
N\left(r_{c}\right) d r_{c} \propto r_{c}^{-\kappa} d r_{c}
$$

The studies summarized by Harris \& Pudritz (1994) yield $\kappa \simeq 3.3 \pm 0.3$.

We can relate the mass and radius distributions by noting that

$$
N\left(M_{c}\right) d M_{c}=N\left(r_{c}\right)\left(\frac{d r_{c}}{d M_{c}}\right) d M_{c}
$$

Assuming a mass-radius relation of the form $M_{c} \propto r_{c}^{x}$, it follows that

$$
N\left(M_{c}\right) d M_{c} \propto M_{c}^{(2-\kappa-x) / x} d M_{c}
$$

Comparing equations (2.1) and (2.5) we find:

$$
\beta=\frac{x+\kappa-2}{x}
$$

or

$$
x=\frac{\kappa-2}{\beta-1}
$$

The observed values of $\kappa$ and $\beta$ yield $x \simeq 2.2$. Assuming that the clouds are in pressure equilibrium, this is consistent with the virial value of $x=2$ within the observational uncertainties.

The young and old globular clusters do not exhibit a significant mass-radius relationship, therefore we can not relate their mass and radius distributions as we did for GMCs. That is, $d r_{*} / d M_{*}=0$, so there is no useful analog to equation (2.4) for globular clusters. However, it is still interesting to compare the radius distributions of globular clusters and GMCs. As before, we express the radius distribution of clusters in the standard way:

$$
N\left(r_{*}\right) d r_{*} \propto r_{*}^{-\eta} d r_{*}
$$

For old globular clusters, $r_{*}$ is most usefully interpreted as the half-light radius, $r_{1 / 2}$, since this radius 
has been shown to be resilient to dynamical evolution effects (e.g. Spitzer \& Thuan 1972; van den Bergh et al. 1991 and references therein). However, a direct comparison between the radius distribution of globular clusters and GMCs is difficult to interpret, even if $r_{1 / 2}$ is used in the comparison (see also Section 4 below). Part of the difficulty stems from the fact that the range of cluster radii in young and old systems is less than the range in mass, so radius distributions are more uncertain than mass distributions. Further, the radii of globular clusters and GMCs are determined in different ways. Finally, the density profiles of GMCs are much shallower than those of globular clusters, so significant evolution of the density profile, and thus the cluster radius, is likely to occur during the cluster formation process. We return to this point in Section 4.

With these caveats in mind, we find that for clusters of large radii the radius distribution of old Milky Way globulars and the young clusters in NGC 3256 can be fit by a power law with $\eta \simeq 3.4$. In these calculations we used the observational data of Zepf et al (1999) for the NGC 3256 clusters and the McMaster catalog (Harris 1996) for Milky Way globular clusters. This slope is consistent with that of the radius distribution of GMCs quoted above. However, the similarity between GMC and globular cluster radius distributions only appears to apply to objects with large radii. Ashman \& Zepf (1998) showed that the old globular clusters of the Milky Way have a half-light radius distribution that exhibits a distinct peak when plotted logarithmically. There is currently only limited information on the radii of young globular clusters, with the most complete study being that of the NGC 3256 system (Zepf et al. 1999). These young clusters also exhibit a peak in the logarithmic radius distribution. A similar peak appears to be present in the young cluster system of the Antennae (Whitmore et al. 1999). There is no evidence for such a peak in the logarithmic radius distribution of GMCs down to the smallest scales that have been observed (Elmegreen \& Falgarone 1996 and references therein).

In terms of understanding globular cluster formation, the differences between the radius distributions of globular clusters and GMCs may be more important than the similarities. Recall that the mass distributions of GMCs and young globular clusters are both well-approximated by single power laws; it is only the old globular clusters that show a turnover in their logarithmic mass distributions. This suggests that the physical processes responsible for the mass distributions of old globular clusters operate on long timescales. In contrast, differences between the radius distributions of GMCs and globular clusters are already apparent in young globular cluster systems, although the data are currently limited. If these results are confirmed by subsequent observations, they will suggest that the deviation from a simple power law of globular cluster radius distributions is produced by processes occurring when the clusters are young or still forming.

\section{The Viability of Molecular Clouds in Merg- ers and Starbursts as Globular Cluster Precursors}

One additional piece of evidence that motivates the scenario discussed in this paper is that young globular clusters are found in mergers and starbursts, but not in the disks of less active star-forming galaxies (with some possible exceptions; see Larson \& Richtler 1999). This suggests that in order to understand the physical conditions that give rise to globular cluster formation, one should examine differences between the conditions in the ISM of starbursts and that of ordinary disk galaxies. One notable difference is that the ISM pressure in galaxy mergers is likely to be several orders of magnitude higher than in the Milky Way. This is expected based on the results of simulations (e.g. Mihos \& Hernquist 1996) and seen in observations (e.g. Heckman, Lehnert \& Armus 1993; Heckman, Armus \& Miley 1990). In this section we show that these higher pressures lead to molecular clouds with properties appropriate for globular cluster precursors.

We noted above that the mass function of GMCs in the Milky Way and other galaxies has a slope which is similar to that observed in young globular clusters in galaxy mergers and the high-mass end of the mass spectrum of old globular clusters. Harris \& Pudritz (1994) defined a "median" mass of Milky Way GMCs such that half the mass in GMCs is in clouds with masses exceeding this value. This median mass is dependent on the slope of the GMC mass function and the assumed upper mass limit of clouds, but for characteristic values such as those used by Harris \& Pudritz (1994) is around $3 \times 10^{5} M_{\odot}$. This is around 2 to 4 times higher than the median mass of old Milky Way globulars. If such GMCs fragmented into bound 
star clusters with a high star formation efficiency of around $25 \%$ to $50 \%$, they would therefore produce clusters with a mass and mass spectrum consistent with that of young globular cluster systems (and also of old globular cluster systems if dynamical evolution removes low-mass clusters). However, the resulting star clusters would not resemble globular clusters in terms of characteristic radii. The median half-light radius of globular clusters in the Milky Way is $3.0 \mathrm{pc}$, whereas the median radius of GMCs is around $20 \mathrm{pc}$.

Qualitatively, it is apparent that a higher external pressure will produce more compact molecular clouds at a given mass. We can quantify this by utilizing the Ebert-Bonner relations (Ebert 1955; Bonner 1956; see also Harris \& Pudritz 1994 and McLaughlin \& Pudritz 1996) for self-gravitating, pressure-bounded isothermal spheres. These may be written as:

$$
\begin{aligned}
M_{c} & =\frac{3.45}{\gamma^{3 / 2}} \frac{\sigma^{4}}{\left(G^{3} P_{s}\right)^{1 / 2}} \\
r_{c} & =\frac{0.69}{\gamma^{1 / 2}} \frac{\sigma^{2}}{\left(G P_{s}\right)^{1 / 2}}
\end{aligned}
$$

where $M_{c}$ and $r_{c}$ refer to the mass and radius of the cloud as before, $P_{s}$ is the surface pressure of the cloud, $\gamma$ is a factor of order unity which is dependent on the nature of the equilibrium, $\sigma$ is the one-dimensional velocity dispersion within the cloud, and $G$ is the gravitational constant. These equations lead to the scaling relation

$$
r_{c} \propto M_{c}^{1 / 2} P_{s}^{-1 / 4}
$$

This scaling can be simply understood by expressing the virial theorem in terms of temperature $T \propto$ $M_{c} / r_{c}$, and noting that density $\rho \propto M_{c} / r_{c}^{3}$ and (assuming that the clouds are in pressure equilibrium) $P_{s} \propto \rho T$.

The Ebert-Bonnor relations are believed to provide a reasonable quantitative description of GMCs in the Milky Way (e.g., Elmegreen 1989). Therefore, the scaling relation (3.3) allows us to calculate the equilibrium properties of GMCs in the high pressure environment of the ISM in a merger, assuming that GMCs in such an environment are in virial equilibrium. If we consider the median Milky Way GMC with a radius of $20 \mathrm{pc}$ and increase the surface pressure by 100 we find the radius of the cloud to be $6.3 \mathrm{pc}$. Given that this is the total radius of the cloud, and that the median half-light radius of old Milky Way globulars (and young globulars in mergers) is around $3.0 \mathrm{pc}$, it is immediately apparent that our compressed GMC has properties consistent with those of a protoglobular cloud. More generally, the above equations imply that in the high-pressure ISM of starbursts any pressure-supported, virialized molecular cloud with a mass comparable to that of a globular cluster will also have a radius characteristic of a globular cluster. This is not the case for the much lower pressures in the ISM of the Milky Way in which the GMCs are much less dense than typical globular clusters.

It is important to note in this context that there is little direct information about the nature of GMCs in starbursts. It is possible that in merger-driven starbursts the GMCs are simply those from the ISM of the progenitor spirals. In this case, the above calculation indicates that such GMCs are viable globular cluster precursors, provided they reach pressure equilibrium with the high-pressure starburst ISM before they fragment to stars. It is also possible that some or all of the GMCs in starbursts form within the highpressure ISM. In this case, the clouds form with radii like those of globular clusters. Irrespective of these details, the critical point is that any viriliazed molecular cloud with a mass characteristic of a globular cluster will also have a radius appropriate for a globular cluster, provided the molecular cloud is in a high-pressure environment like the ISM of starbursts.

\subsection{The Star Formation Efficiency in Com- pressed GMCs}

A general requirement for the formation of any bound star cluster is that the star formation efficiency must be locally high: somewhere around $25 \%$ to $50 \%$. If we consider a model in which protoglobular clouds are compressed GMCs with masses like those in the Milky Way, the fact that the median mass of GMCs is a factor of 2 to 4 greater than that of globular clusters implies a global star formation efficiency around this value is required to produce globular clusters. We regard it as promising that the star formation efficiency required by both arguments is the same. It is also promising that the starburst/merger environment in which GMCs might be compressed is observed to have a higher efficiency of star formation overall. The physical connection between high pressure and high star formation efficiency has been discussed by several authors (notably Jog \& Solomon 1992; Jog \& Das 1996; Elmegreen and Efremov 1997).

The critical question is how to achieve such a high star formation efficiency in a GMC. A notable feature 
of a compressed GMC is that its dynamical timescale of around $10^{6}$ years is very short (see also Elmegreen \& Efremov 1997). This is comparable to the timescale of various disruptive processes associated with massive stars such as ionization fronts, stellar winds, and supernova explosions. These processes have the potential to terminate star formation in GMCs and, if this results in a low star formation efficiency, may lead to the resulting star cluster becoming unbound. It is therefore plausible that within a compressed GMC the short dynamical timescale will allow a large fraction of the original gas mass to be converted into stars. In other words, clusters forming from high density GMCs do so with a high star formation efficiency. On the other hand, in lower density GMCs such as those in the low-pressure ISM of the Milky Way, disruptive processes associated with massive stars may regulate or terminate star formation before much fragmentation has occurred.

We can investigate the feasibility of this idea by considering the scaling of dynamical time of virialized clouds with a range of external pressures. For clouds with constant density as a function of radius, the dynamical time may be written as:

$$
t_{d}=\left(\frac{3 \pi}{32 G \rho}\right)^{1 / 2}=\left(\frac{\pi^{2} r_{c}^{3}}{8 G M_{c}}\right)^{1 / 2}
$$

We can now use the Ebert-Bonner relations to eliminate $r_{c}$. This yields:

$$
M_{c} \simeq 2 \times 10^{3} \gamma^{-3 / 2}\left(\frac{t_{d}}{10^{6} \mathrm{yr}}\right)^{4}\left(\frac{P_{s}}{10^{5} \mathrm{k} \mathrm{cm}^{-3} \mathrm{~K}}\right)^{3 / 2}
$$

where $M_{c}$ is in units of solar masses. $P_{s}$ is normalized to a value appropriate to GMCs in the Milky Way, which is believed to be about a factor of five greater than the typical ISM pressure because of the HI envelopes surrounding GMCs (Elmegreen 1989; see also Harris \& Pudritz 1994).

We can use the above relation to estimate the maximum mass of Ebert-Bonner clouds that, at a given surface pressure, have a dynamical time less than $10^{6}$ years. For surface pressures around the $10^{5} k \mathrm{~cm}^{-3} \mathrm{~K}$ characteristic of GMCs in the Milky Way, the maximum cloud mass satisfying this requirement is around $10^{3} M_{\odot}$. For surface pressures around $10^{7}-10^{8} k \mathrm{~cm}^{-3} \mathrm{~K}$ expected for clouds in the ISM of starbursts, this mass increases to around $10^{7} M_{\odot}$. Although this is clearly a simplified calculation, it illustrates an important trend. Specifically, higher ex- ternal pressures lead to an increase in the maximum cloud mass for which the dynamical time of the cloud is less than timescales characteristic of disruptive stellar processes. Despite this simplification, however, the central idea that GMCs in a high-pressure environment are denser and therefore fragment more rapidly will still apply in a more realistic treatment.

Finally, it is worth noting in this context that the possibility of rapid fragmentation and star formation is highly pertinent to one of the most striking observational characteristics of globular clusters: the starto-star homogeneity in the abundance of iron-peak elements. If star formation occurs on a timescale comparable to or less than the lifetime of massive stars, metals from supernovae will not end up in low-mass globular clusters stars observed today. In this case, the only effect of supernovae will be to purge young globular clusters of gas, which is required anyway. This point has been made by Elmegreen and Efremov (1997) in the context of their model of cluster formation.

\section{From Clouds to Clusters: A Variable Star Formation Efficiency}

The results summarized in Section 2 lead to the important conclusion that any model of globular cluster formation (and cluster formation in general) needs to explain the difference between the mass-radius relation of star clusters and their precursor clouds. In other words, an essential ingredient of any globular cluster formation model is a mechanism which modifies the virial mass-radius relation of the original clouds.

In this section we investigate whether a variable star formation efficiency can produce the observed mass-radius relation of clusters forming from GMCs. Part of the motivation for this approach is that, as noted in Section 3, if GMCs are the precursors to globular clusters, the star formation efficiency must be much higher in GMCs in the high-pressure ISM of starbursts than in GMCs in the disk of the Milky Way. The question is therefore whether a variable star formation efficiency can wipe out the mass-radius relation of GMCs and produce cluster systems with properties consistent with the observed distributions of globular cluster masses and radii.

In the following calculations we investigate the consequences for the mass-radius relation of clusters of a variation in the star formation efficiency with: (i) the 
binding energy per unit mass of clouds; (ii) the density of clouds. In both cases, we define $\epsilon$ to be the star formation efficiency such that

$$
\epsilon=\frac{M_{*}}{M_{c}}
$$

where $M_{*}$ is the mass of the star cluster resulting from a cloud mass $M_{c}$.

To determine the mass-radius relation of clusters, it is clear that we also need to be able to calculate the final cluster radius, $r_{*}$, produced by a cloud with radius $r_{c}$. For any star formation efficiency less than unity one expects that $r_{*}>r_{c}$, with lower efficiencies producing larger (and possibly unbound) clusters. We assume that a cloud fragments and produces an initial cluster of radius $r_{c}$. If the gas loss is slow (i.e., it occurs on timescales longer than the cluster dynamical time) the product of mass and radius is an adiabatic invariant. Under these conditions, the final cluster radius is related to the star formation efficiency by:

$$
\frac{r_{*}}{r_{c}} \simeq \epsilon^{-1} .
$$

(Hills 1980; Richstone \& Potter 1982; Mathieu 1983). This expression has recently been verified numerically by Geyer \& Burkert (2001) for the case of slow mass loss. For more rapid mass loss, these authors find larger expansion rates at a given $\epsilon$, but for $\epsilon<0.4$ the clusters are unbound.

While the dependence of cluster expansion on star formation efficiency seems well established, there are a few complications in directly comparing the radii of clusters with the radii of their precursor clouds. We have assumed above that the precursor cloud fragments to a cluster which initially has the same radius as the cloud, $r_{c}$. However, the density profiles of GMCs and globular clusters have a different slope and form, with GMCs being described by shallow powerlaws and most globular clusters being well-fit by a steeper King profile. There is growing evidence that young star clusters have power-law density profiles, but they are still steeper than those of GMCs. For example, density profiles inside GMCs are typically around $R^{-2}$ to $R^{-1}$, with clumps inside clouds having even shallower profiles (Elmegreen \& Falgarone 1996). In young LMC clusters there is a range of power-law slopes with $R^{-2.5}$ being typical (Elson et al 1987), while Whitmore et al (1999) find a slope consistent with this value for a very young (6-8 Myr) globular cluster in the merger NGC 4038/9. This difference in slope between the density profiles of clouds and clusters means that their must be some evolution of the density profile, and thus the radius, before or during the fragmentation of a cloud into a cluster. This issue is compounded by the fact that the observed radii of clouds and globular clusters are determined in different ways, with the cluster radius usually being a half-light radius. Finally, the details of cloud fragmentation may be important in determining the final cluster radius. For example, it is possible that fragmentation in the center of a GMC leads to contraction of the remaining gaseous cloud before these regions fragment.

\subsection{Star formation efficiency variation with cloud binding energy}

The binding energy per unit mass of a self-gravitating system scales as $M / r$. If we assume that the star formation efficiency varies as some power of binding energy per unit mass of the progenitor clouds, we can write

$$
\epsilon \propto\left(\frac{M_{c}}{r_{c}}\right)^{n}
$$

From equation (2.3) it follows that

$$
\epsilon \propto M_{c}^{n / 2} P_{s}^{n / 4}
$$

and thus

$$
M_{*} \propto M_{c}^{(n+2) / 2} P_{s}^{n / 4}
$$

Equations (3.3) and (4.2) yield:

$$
r_{*} \propto r_{c}^{(1-n)} P_{s}^{-n / 2}
$$

Equations (4.5) and (4.6) can be used, along with equation (3.3), to obtain a mass-radius relation for the resulting clusters:

$$
r_{*} \propto M_{*}^{(1-n) /(n+2)} P_{s}^{[1-2(n+1)] /[2(n+2)]}
$$

Note that for $n=0$ these expressions give $M_{*} \propto M_{c}$, $r_{*} \propto r_{c}$, and $r_{*} \propto M_{*}^{1 / 2} P_{s}^{-1 / 4}$ as expected.

The weak or absent mass-radius correlation of globular clusters is reproduced if the exponent on $M_{*}$ in (4.7) is close to zero. This occurs when $n \simeq 1$. For the specific case of the young globular clusters in NGC 3256, Zepf et al (1999) found $r_{*} \propto M_{*}^{0.1 \pm 0.1}$ (assuming a constant cluster mass-to-light ratio) which is reproduced by $n \simeq 0.75 \pm 0.25$. The value $n=1$ is interesting since it corresponds to the case of a star formation efficiency which is directly proportional to 
the binding energy per unit mass of the precursor gas clouds (see also Section 5 below). An increase in star formation efficiency with velocity dispersion (which scales as the square root of binding energy per unit mass) has been suggested by Elmegreen et al (1993) and Elmegreen \& Efremov (1997).

Equation (4.5) also allows us to investigate the variation in star formation efficiency, and thus the upper mass of clusters, with pressure. This is important because, as noted in Section 1, we require a much higher star formation efficiency in GMCs in the high-pressure ISM of starbursts than occurs in GMCs in the Milky Way. Since the above analysis only provides scaling relations, we need to normalize the relations to investigate this question quantitatively. We suppose that $\epsilon$ reaches a maximum value of 0.5 for $M_{c}=10^{7} M_{\odot}$ and $P_{s}=10^{8} \mathrm{k} \mathrm{cm}^{-3} \mathrm{~K}$. The values are somewhat arbitrary, corresponding to a characteristic cloud surface pressure in the ISM of starbursts and a cloud mass sufficient to produce a high-mass globular cluster. Thus, for $n=1$, (4.5) becomes:

$$
\frac{M_{*}}{M_{c}}=0.5\left(\frac{M_{c}}{10^{7} M_{\odot}}\right)^{1 / 2}\left(\frac{P_{s}}{10^{8} k \mathrm{~cm}^{-3} \mathrm{~K}}\right)^{1 / 4}
$$

Thus the efficiency of star formation in the Milky Way ISM with $P_{s}=10^{5} \mathrm{k} \mathrm{cm}^{-3} \mathrm{~K}$ is around 0.1 for $M_{c}=10^{7} M_{\odot}$ and 0.01 for $M_{c}=10^{5} M_{\odot}$. While these efficiencies are somewhat higher than observed, they do suggest that the formation of massive, bound star clusters is much less likely in the ISM of the Milky Way than in the ISM of starbursts.

\subsubsection{Constraints on the scenario}

Important constraints on the dependence of star formation efficiency on cloud properties are provided by observations of the cloud and cluster masses and mass distributions. We relate the mass distributions of clouds and clusters by

$$
N\left(M_{*}\right) d M_{*} \propto N\left(M_{c}\right)\left(\frac{d M_{c}}{d M_{*}}\right) d M_{*}
$$

A variable star formation efficiency will, in general, lead to different slopes for the cloud and cluster mass distributions. Equations (4.5) and (4.9) can be used to quantify the difference in slope:

$$
\alpha=\frac{2 \beta+n}{n+2} .
$$

(For $n=0, \alpha=\beta$ as expected.) Alternatively,

$$
n=\frac{2(\beta-\alpha)}{\alpha-1}
$$

Equation (4.11) allows us to use the observed values of $\alpha$ and $\beta$ to constrain the permitted range of $n$. Since $\alpha$ and $\beta$ are comparable, it is apparent that small values of $n$ are favored. For example, for the mass function slopes given in Section 2 of $\beta=2$ and $\alpha=1.8$ one obtains $n=0.5$, giving a mass-radiius relation consistent with that of the young clusters in NGC 3256. The observational uncertainty on $\alpha$ and $\beta$ means that $n=1$ is marginally consistent with the data.

Note that if a variable star formation efficiency of this form is responsible for wiping out the cloud massradius relation, it is inevitable that the cluster mass function is shallower than the precursor cloud mass function. In other words, our picture predicts that $\alpha<\beta$. Thus determinations of the distributions of cloud and cluster mass have the potential to refute or support this scenario. Unfortunately, the current uncertainties in these quantities, as well as the fact that cluster and cloud mass spectra are rarely derived for the same systems, do not allow a definitive test of the scenario. However, future observations of the mass functions of molecular clouds and globular clusters may be able to strongly constrain the dependence of star formation efficiency on binding energy.

\subsection{Star formation efficiency variation with cloud density}

Another case of potential interest is a variation of star formation efficiency with cloud density. If we suppose that $\epsilon$ has a power-law dependence on density, we can write:

$$
\epsilon \propto \rho^{m} \propto\left(\frac{M_{c}}{r_{c}^{3}}\right)^{m}
$$

Following the same treatment as in Section 4.1 we obtain the following relations:

$$
\begin{aligned}
& \epsilon \propto M_{c}^{-m / 2} P_{s}^{3 m / 4} \\
& M_{*} \propto M_{c}^{(2-m) / 2} P_{s}^{3 m / 4} \\
& r_{*} \propto r_{c}^{(1+m)} P_{s}^{-m / 2} \\
& r_{*} \propto M_{*}^{(1+m) /(2-m)} P_{s}^{[3-(m+1) 4] /[2(2-m)]}
\end{aligned}
$$

Again, the behavior of these expressions when $m=0$ is as expected. 
The exponent on $M_{*}$ in this relation can not equal zero for positive $m$. Thus even though relationships like the Schmidt law $\left(\epsilon \propto \rho^{2}\right)$ provide a good description of global star formation efficiency, such a relationship can not wipe out the mass-radius relation of star clusters forming from GMCs, nor any other system of pressure-supported progenitor clouds obeying the virial theorem. The failure of the widely applied Schmidt law and similar relationships between star formation efficiency and density to account for the observation that cluster mass and radius are independent demonstrates the importance of this observation for constraining globular cluster formation models.

\subsection{Pressure variations in the ISM}

The above discussion of the effects of a variable star formation efficiency on the mass-radius relation of globular clusters implicitly assumes a constant ISM pressure. Thus for a given cloud mass, the cloud radius and resulting cluster radius is uniquely determined. In practice, it seems inevitable that the ISM pressure in starbursts exhibits some variation with location. One immediate consequence of including such pressure variations is that the precursor GMCs occupy a band rather than a line in the mass-radius plane. In the context of our scenario, this means that clouds at a given mass will produce globular clusters with a range of densities. A large range in density at a given mass is a notable feature of the globular cluster system of the Milky Way. The same broad distribution of densities appears to be the case for young globular clusters in NGC 3256. It is currently difficult to link quantitatively the pressure variations in the ISM and the observed density range of young and old globular cluster systems. Old globular clusters have likely undergone significant mass-loss, while the densities of young globular clusters are not well determined. Future observations of young globular cluster systems and the pressure in the surrounding ISM may produce useful constraints on the relation between these quantities.

An additional issue is whether pressure variations and the resulting dispersion in the GMC mass-radius relation have any impact on the mass-radius relation of the resulting clusters derived above. We have carried out a preliminary study of this question using Monte Carlo simulations that generate an ensemble of clouds with a mass distribution like that observed for GMCs. Each cloud is randomly assigned a pressure drawn from a log-normal distribution with a peak value consistent with that of the ISM in starbursts. The dispersion of this log-normal distribution was varied with simulations for one, two and three dex performed. Individual cloud radii are calculated from the assigned mass and pressure of each cloud using the Ebert-Bonner relations (3.1) and (3.2). The final globular cluster masses and radii are obtained by using one of the prescriptions for star formation efficiency described above.

We have found that pressure variations alone can not wipe out the virial mass-radius relation of the precursor clouds. That is, if we assume a constant star formation efficiency, the resulting clusters still exhibit the scaling relation $r \propto M^{0.5}$ of the GMCs. We have also used this technique to study the effects of pressure variations for the case of a star formation efficiency that varies linearly with cloud binding energy per unit mass (i.e., the case $n=1$, see Section 4.1). In this case, the dispersion in the mass-radius relation produced by pressure variations has the effect of slightly weakening constraints derived for the constant pressure situation. This is simply because the dispersion in the mass-radius relation produces an uncertainty in the measured slope of the mass-radius relation, at least for a finite sample size. To quantify this somewhat, recall that in the constant pressure case a relation of the form $r_{*} \propto M_{*}^{y}$ is produced with $y$ being dependent on $n$. Our simulations suggest that pressure variations of the order described above will produce cluster masses and radii that are indistinguishable from no mass-radius relation if $y$ is less than about 0.1 , given a sample size comparable to the number of young clusters observed in NGC 3256.

\section{Discussion}

Observational advances over the last decade mean that theories of globular cluster formation can now be motivated and constrained by observation. We have argued in this paper that the observed formation of globular clusters in starbursts and mergers reveals the conditions under which globular cluster formation occurs. We have shown that the high-pressure of the ISM of mergers and starbursts implies that GMCs in this environment have the right properties to be globular cluster precursors, if they form stars with high efficiency. A high star formation efficiency in these dense GMCs may be a natural consequence of their short dynamical timescales and high binding energies. However, all GMCs studied to date follow a strong 
scaling relation between mass and radius, whereas the correlation between mass and radius for globular clusters appears to be weak or non-existent. We have suggested that a star formation efficiency that is a function of cloud binding energy can wipe out the mass-radius relation of the progenitor GMCs. Such a variation automatically implies a much higher star formation efficiency in GMCs in the high-pressure environment of starbursts than in the low-pressure ISM of ordinary disk galaxies like the Milky Way.

There are plausible reasons why star formation efficiency might be roughly proportional to the binding energy per unit mass of clouds (see also Elemegreen et al 1993; Elmegreen and Efremov 1997). To a first approximation, the disruptive energy input from massive stars will be proportional to the number of such stars and thus the mass of the cloud, hence the normalization of binding energy to unit mass. While the details of the disruption produced by massive stars is beyond the scope of this paper, it seems likely that clouds with a higher binding energy will be less affected by such disruption and therefore convert a higher fraction of their gas mass into stars. We plan to address this question with simulations in a future paper.

A key ingredient of our scenario stems from the observation that the mass and radius distributions of GMCs and globular clusters are similar, whereas their mass-radius relations are not. This observation constitutes a challenge for any scenario in which GMCs (or other virialized gas clouds) are globular cluster progenitors. Specifically, it is difficult to eliminate the original mass-radius relation without significantly altering either the mass distribution or the radius distribution. We have shown that a star formation efficiency that varies with cloud binding energy leads to the testable prediction that the globular cluster mass distribution is shallower than that of the clouds. Constraints based on the radius distribution of clouds and clusters are more difficult to apply. This is partly because the radius distribution of GMCs is a simple power-law whereas that of young clusters is not. Thus it is not possible to directly compare the slopes of cloud and young cluster power-laws and obtain constraints on the scenario as we did for the mass distributions. Other complicating factors include the difference in the density profiles of clouds and clusters (suggesting the radius changes as the density profile steepens from the cloud to cluster slope) and the fact that cloud and cluster radii are determined in differ- ent ways.

We note that the expansion of clusters due to adiabatic mass loss has been exploited by other authors in an attempt to understand the properties of old globular clusters (e.g. Gunn 1980; Djorgovski 1991; Djorgovski \& Meylan 1994). However, these previous studies assumed a different mass-radius relation for the "initial" clusters. More importantly, in the present scenario the "lost" mass is simply gas mass that is not converted into stars. The studies mentioned above assume stellar mass-loss is responsible for cluster expansion and an evolution of the massradius relationship. The observation that young cluster systems do not follow the mass-radius relation of the precursor clouds tends to favor early loss of mass from the clusters, as envisioned in our scenario.

While our picture addresses the formation of globular clusters at the current epoch in mergers and starbursts, it does not directly address the formation of old globular clusters found around galaxies from dwarfs to massive spirals and ellipticals. We have previously argued (Ashman and Zepf 1992) that many of the old globular clusters around ellipticals formed in mergers at earlier epochs. As such, the formation of these globular clusters is directly analogous to those forming in mergers at the current epoch and addressed in this paper. In a future paper we will investigate whether the physical conditions that give rise to globular clusters in starbursts and mergers are present in young dwarf galaxies and spirals.

We are grateful to useful discussions with Dean McLaughlin and Richard Larson. This work was supported in part by NASA Astrophysics Theory Grant NAG5-9168.

\section{REFERENCES}

Ashman, K.M. 1990, MNRAS, 247, 662

Ashman, K.M., \& Zepf, S.E. 1992, ApJ, 384, 50

Ashman, K.M., \& Zepf, S.E. 1998, Globular Cluster Systems (Cambridge University Press: Cambridge).

Bonner, W.B. 1956, MNRAS, 116, 351

Burkert, A., \& Smith, G.H. 2000, ApJ, 542, L95

Carlson, M.N. et al. 1998, AJ, 115, 1778

Djorgovki, S.G. 1991, in ASP Conf. Ser., Vol. 13. Formation and Evolution of Star Clusters, ed. K. Janes (San Francisco: ASP), 112 
Djorgovki, S.G. \& Meylan, G. 1994, AJ, 108, 1292

Ebert, R. 1955, Z. Astrophys, 37, 222

Elmegreen, B.G. 1989, ApJ, 338, 178

Elmegreen, B.G., \& Efremov, Y.N. 1997, ApJ, 480, 235

Elmegreen, B.G., \& Falgarone, E. 1996, ApJ, 471, 816

Elmegreen, B.G., Kaufman, M., \& Thomasson, M. 1993, ApJ, 412, 90

Elson, R.A.W., Fall, S.M., \& Freeman, K.C. 1987, ApJ, 323, 54

Fall. S.M., \& Rees, M.J. 1985, ApJ, 298, 18

Geyer, M.P., \& Burkert, A. 2001, MNRAS, 323, 988

Gunn, J.E. 1980, in Globular Clusters, ed. D. Hanes \& B. Madore (Cambridge: Cambridge Univ. Press), 301

Harris, W.E. 1991, ARAA, 29, 543

Harris, W.E. 1996, AJ, 112, 1487

Harris, W.E., \& Pudritz, R.E. 1994, ApJ, 429, 177

Heckman, T.M., Armus, L., \& Miley, G.K. 1990, ApJS, 74, 833

Heckman, T.M., Lehnert, M.D., Armus, L. 1993, in The Environment and Evolution of Galaxies, ed. J.M. Shull \& Thronson, H.A., Jr. (Dordrecht: Kluwer), 455

Hills, J.G. 1980, ApJ, 225, 986

Jog, C., \& Das, M. 1996, ApJ, 473, 797

Jog, C., \& Solomon, P. 1992, ApJ, 387, 152

Johannson, L.E.B. 1991, in IAU Symp. 146, Dynamics of Galaxies and Their Molecular Cloud Distributions, ed. F. Combes \& F. Casoli, (Dordrecht: Kluwer), 1

Larsen, S.S., \& Richtler, T. 1999, A\&A, 345, 59

Larson, R.B. 1981, MNRAS, 194, 809

Mathieu, R.D. 1983, ApJ, 267, L97

McLaughlin, D.E., \& Pudritz, R.E. 1996, ApJ, 457, 578

McCrea, W.H. 1982, in Progress in Cosmology, ed. A.W. Wolfendale (Reidel: Dordrecht), 239

Mihos, C.M. \& Hernquist, L. 1996, ApJ, 464, 641

Miller, B.W., Whitmore, B.C., Schweizer, F., \& Fall, S.M. 1997, AJ, 114, 2381

Moore, B. 1996, ApJLett, 416, L13
Murray, S.D., \& Lin, D.N.C. 1992, ApJ, 400, 265

Peebles, P.J.E., \& Dicke, R.H. 1968, ApJ, 154, 891

Richstone, D.O., \& Potter, M.D. 1982, ApJ, 254, 451

Rubio, M., Lequeux, J., \& Boulanger, F. 1993, A\&A, 271,9

Schweizer, F. 1998, in Galaxies: Interactions and Induced Star Formation, Saas-Fee Course 26, eds. R.C. Kennicutt et al. (Springer: Berlin), 105

Spitzer, L. \& Thuan, T.X. 1972, ApJ, 175, 31

Testi, Palla, \& Nota 1999, A\&A, 342, 515

van den Bergh, S. 1991, ApJ, 369, 1

van den Bergh, S., Morbey, C.L., \& Padzer, J. 1991, ApJ, 375, 594

Vesperini, E. 1997, MNRAS, 287, 915

Whitmore, B.C., \& Schweizer, F. 1995, AJ, 109, 960

Whitmore, B.C., Zhang, Q., Leitherer, C., Fall, S.M., Schweizer, F., \& Miller, B.W. 1999, AJ, 118, 1551

Wilson. C.D., \& Scoville, N. 1990, ApJ, 363, 435

Zepf, S.E., Ashman, K.M., English, J., Freeman, K.C., \& Sharples, R.M. 1999, AJ, 118, 752

Zhang, Q., \& Fall, S.M. 1999, ApJ, 527, 81

Zinn, R. 1985, ApJ, 293, 424

This 2-column preprint was prepared with the AAS LATEX macros $\mathrm{v} 4.0$. 